\title{
Precision Mechanical Measurement Using the Levitation Mass Method (LMM)
}

\author{
Yusaku Fujii, Tao Jin and Koichi Maru \\ Department of Electronic Engineering, Gunma University, \\ 1-5-1 Tenjin-cho, Kiryu, Gunma, 376-8515, JAPAN \\ e-mail: fujii@el.gunma-u.ac.jp
}

Received 22 October 2010, Accepted 23 November 2010

\begin{abstract}
The present status and the future prospects of a method for precision mass and force measurement, the levitation mass method (LMM), are reviewed. The LMM has been proposed and improved by the authors. In the LMM, the inertial force of a mass levitated using a pneumatic linear bearing is used as the reference force applied to the objects under test, such as force transducers, materials or structures. The inertial force of the levitated mass is measured using an optical interferometer. The three typical applications of the LMM, i.e. the dynamic force calibration, the micro force material tester and the space scale, are reviewed in this paper.
\end{abstract}

Keywords: Dynamic calibration, Impact force, Dynamic force, Force sensor, Force Transducer, Inertial force, inertial mass, Mass measurement, Material tester, Micro force, Optical interferometer, The International Space Statioin, Levitation Mass Method

\section{Introduction}

Force is one of the most basic mechanical quantities and is usually measured using force transducers. However, the dynamic calibration method of force transducers has not been established. It is due to two major problems concerning material testing. On the one hand, it is difficult to evaluate the uncertainty of the measured value of the varying force. On the other hand, it is difficult to evaluate the uncertainty of the time at which the varying force is measured. According to this, "Levitation Mass Method" (LMM) was proposed by the authors. In this method, the inertial force of a mass, which is levitated using a pneumatic linear bearing, is used as the reference force applied to the objects being tested, such as force transducers, materials and structures. As shown in Figure 1, the inertial force of the levitated mass is measured using an optical interferometer. In the LMM, only the motion-induced time-varying beat frequency is measured during the measurement, and all the other quantities, such as velocity, position, acceleration and force, are numerically calculated afterwards. The results between the obtained quantities are in good synchronism. In addition, force is directly calculated according to its definition, that is, the product of mass and acceleration.

The authors have proposed a method, the Levitation Mass Method (LMM). In the LMM, the inertial force of a mass levitated using a pneumatic linear bearing is used as the reference force applied to the objects under test, such as force transducers, materials or structures. The inertial force of the levitated mass is measured using an optical interferometer. The author has modified it as calibration methods for all the three categories of the dynamic force calibration ${ }^{1-5)}$, that are the dynamic calibration method under impact load ${ }^{1}$, the dynamic calibration method under oscillation $\operatorname{load}^{2}$ and the dynamic calibration method under step load ${ }^{3)}$. The authors have applied the LMM for material testing, such as methods for evaluating material viscoelasticity under an oscillating load" $\mathrm{d}^{6)}$ and under an impact load ${ }^{7,8)}$, a method for evaluating material friction ${ }^{9,10)}$, a method for evaluating biomechanics ${ }^{11,12)}$, a method for evaluating a dynamic performance of a liner motor ${ }^{13)}$, mass measurement device (MMD) for use in the International Space Station (ISS) ${ }^{14-18)}$, a method for evaluating dynamic response of impact hammers ${ }^{19)}$, a method for generating and measuring a micro-Newton level forces ${ }^{20-23)}$. The author has also applied the LMM as a method for investigating the frictional characteristics of pneumatic linear bearings $\mathrm{s}^{24,25)}$ and the linear ball bearing ${ }^{26,27)}$. To improve the efficiency of the LMM, a pendulum mechanism ${ }^{28)}$ for use as a substitute of a pneumatic linear bearing and a frequency measurement technique ${ }^{29-31)}$ using a digitizer instead of an electronic frequency counter have been developed. Recently, a method for correcting the effect of the inertial mass on dynamic force measurements has been proposed based on the $\mathrm{LMM}^{32-34)}$. The optical interferometer has been improved ${ }^{35-38)}$.

In this paper, the recent achievement and the future prospects on the method for precision mass and force measurements, the levitation mass method (LMM) are reviewed. The three typical applications of the LMM, which are shown in Table 1, i.e. the dynamic force calibration, the micro force material tester and the space scale, are given. 


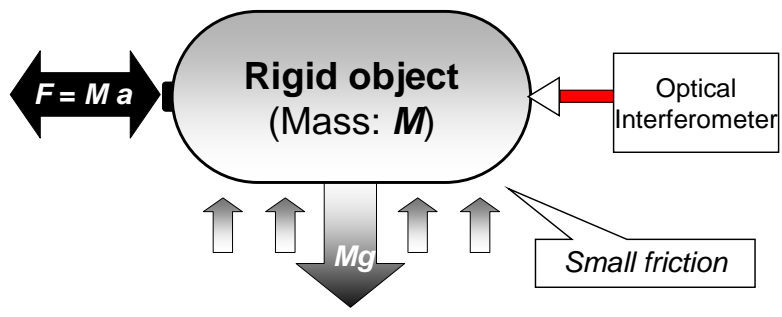

Figure 1. Principle of the Levitation Mass Method (LMM)

Table 1. Applications of the Levitation mass method (LMM)

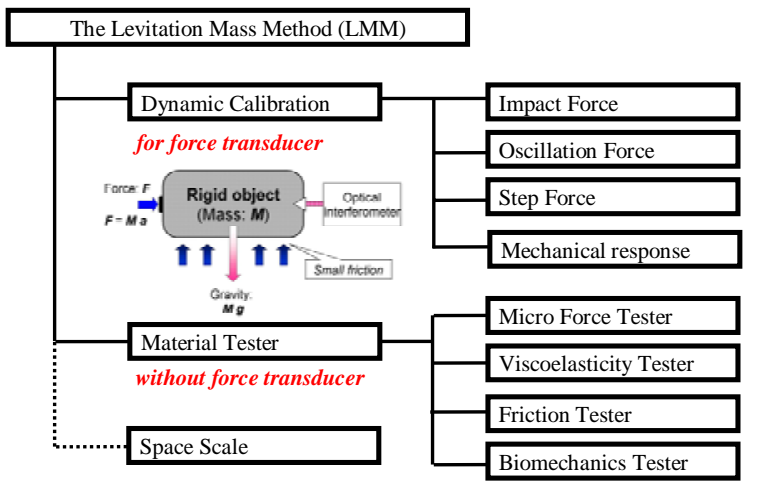

\section{Dynamic Calibration Methods for Force Transducers}

Methods for evaluating the dynamic response of force transducers based on the LMM, which are for the impact $^{1,9,13)}$, oscillation ${ }^{2)}$ and step response evaluation method $^{3)}$ and also for the method of evaluating the electrical and mechanical responses of the force transducer against the impact load ${ }^{24)}$, are reviewed.

\subsection{Impact response calibration method}

Figure 2 shows the experimental setup for measuring the impact force applied on the force transducer under tested. An impulse is generated and applied on the transducer through the moving part which levitation by a pneumatic linear bearing and with small friction to the transducer. An initial velocity is given to the moving part manually. The width and the intensity of the impulse are adjusted by changing the rubber damper and the initial speed of the moving part. The output signal of the force transducer is recorded using digital voltmeter with a sampling interval of $0.2 \mathrm{~ms}$.

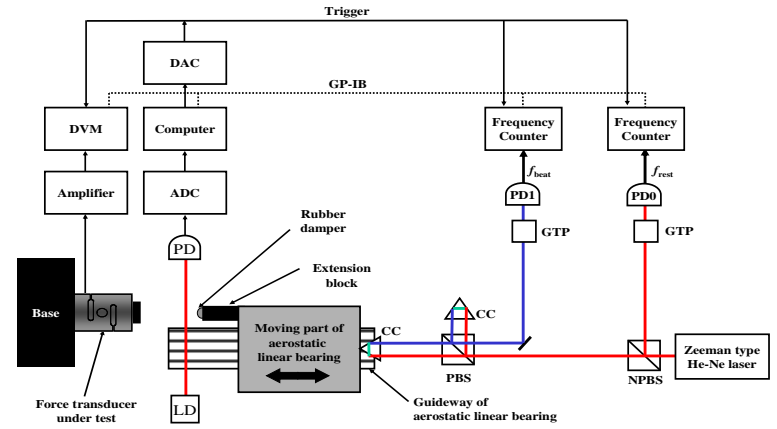

Figure 2. Experimental setup. Code: $\mathrm{CC}=$ cube corner prism, PBS = polarizing beam splitter, $\mathrm{NPBS}=$ non-polarizing beam splitter, GTP = Glan-Thompson prism, $\mathrm{PD}=$ photo diode, $\mathrm{LD}=\mathrm{l}$ aser diode, $\mathrm{ADC}=$ analog-to-digital, DAC = digital-to-analog converter, $\mathrm{PC}=$ computer.

A Zeeman-type two-frequency He-Ne laser is used as the light source of the optical interferometer. The interferometer has two photo-detectors; PD0 and PD1. The frequency difference between the two orthogonal polarization states emitted from the laser, $f_{\text {rest }}$, is monitored using a Glan-Thompson prism (GTP) and the first photo-detector, PD0. The velocity of the mass, v, is measured as the Doppler shift frequency, $f_{\text {Doppler, }}$ which can be expressed as follows:

$$
\begin{aligned}
& \mathrm{v}=\boldsymbol{I}_{\text {air }}\left(\boldsymbol{f}_{\text {Doppler }}\right) / 2, \\
& \boldsymbol{f}_{\text {Doppler }}=-\left(\boldsymbol{f}_{\text {beat }}-\boldsymbol{f}_{\text {rest }}\right),
\end{aligned}
$$

where $\boldsymbol{l}_{\text {air }}$ is the wavelength of the signal beam under the experimental conditions, $\boldsymbol{f}_{\text {beat }}$ is the beat frequency, which is the frequency difference between the signal beam and the reference beam and appears as the beat frequency at PD1, and $\boldsymbol{f}_{\text {rest }}$ is the rest frequency which is the value of $f_{\text {beat }}$ when the moving part of the aerostatic bearing is at a standstill.

The frequency $\boldsymbol{f}_{\text {beat }}$ appearing at PD1 is measured using an electric frequency counter (model: R5363; manufactured by Advantest Corp., Japan). It continuously measures and records the beat frequency, $f_{\text {beat }}, 1000$ times with a sampling interval of $\mathrm{T}=400$ / $f_{\text {beat }}$, and stores the values in its memory. The sampling period of the counter is approximately $0.15 \mathrm{~ms}$ at a frequency of $2.7 \mathrm{MHz}$. The other counter of the same model measures the frequencies $\boldsymbol{f}_{\text {rest }}$ appearing at PD0. From the measured Doppler shift frequency, the velocity, acceleration and inertial force of the mass are calculated.

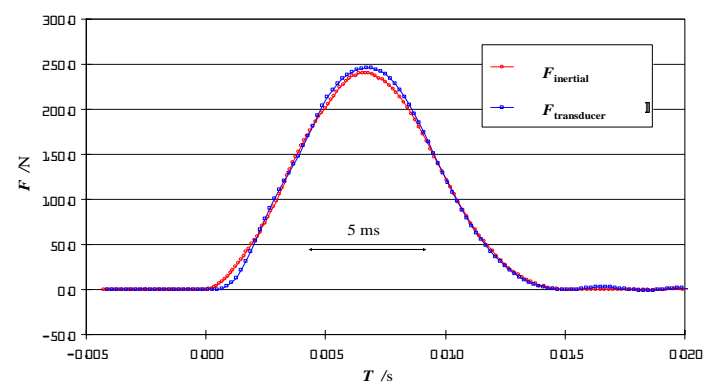

Figure 3. Results of impact force calibration. 
The response of the transducer to an impulse with the half value width of approximately $6.7 \mathrm{~ms}$ is shown in Figure 3. The red curve is the output of interial force. The blue curve is the output signal of the transducer, $\boldsymbol{F}_{\text {transducer, seems to vibrate at its }}$ characteristic frequency during and after the application of the impulse.

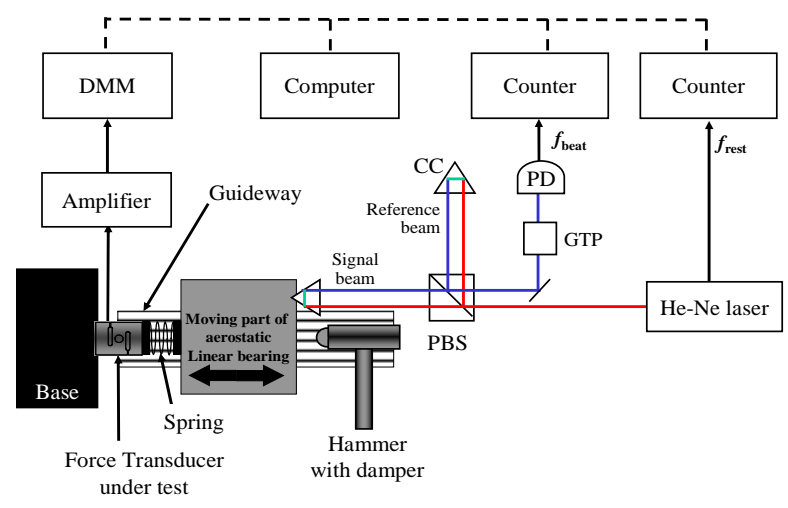

Figure 4. Experimental setup for oscillation force calibration

\subsection{Oscillation response calibration method}

The schematic of the experimental setup for evaluating the continuous oscillation response of force transducers $^{12)}$ is illustrated in Figure 4. The force transducer which connects to a mass using a steel spring is firmly attached to the base. In order to get a linear motion, a pneumatic linear bearing is used. The friction acting on the mass through bearing is very small. The inertial force of the mass is used as a standard oscillation force and compared with the output signal of the force transducer, when the mass-spring system is continuously oscillating. The initial kinetic energy of the spring-mass system is given by manually hitting the mass using a hammer. The inertial force acting on the mass is highly accurately calculated by Newton's second law and measuring the velocity of the mass using an optical interferometer.

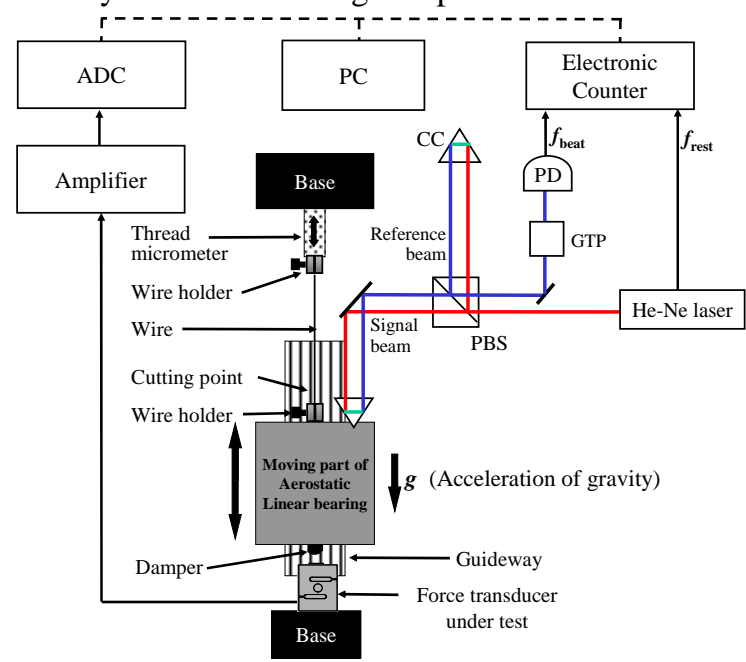

Figure 5. Experimental setup for step force calibration

\subsection{Step response calibration method}

The experimental setup for evaluating the step response of force transducers ${ }^{13)}$ is given in Figure 5. A mass, $\boldsymbol{M} \mathrm{kg}$, just above a force transducer under test, is suspended using a stainless steel wire. The initial distance between the object and the force transducer is adjusted using a thread micrometer attached to the upper base.

To realize a vertical motion with sufficiently small friction, a pneumatic linear bearing is also used. The velocity of the mass is measured highly accurately using an optical interferometer. The measurement procedure is as follows: at the beginning, the object is raised, using the wire, just above the transducer; then the object is dropped onto the transducer by cutting the wire using a wire cutter. A rubber damper is attached to the contact point of the object in order to moderate the steepness of the step force. The inertial force acting on the mass is calculated from the velocity of the mass.

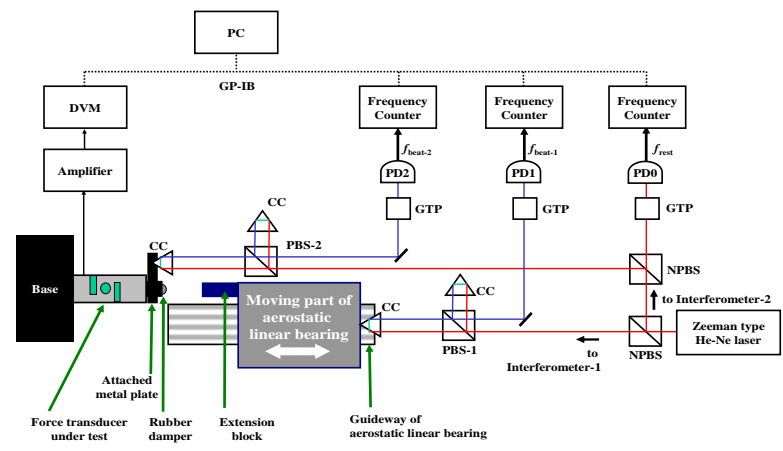

Figure 6. Experimental setup. Code: $\mathrm{CC}=$ cube corner prism, $\mathrm{PBS}=$ polarizing beam splitter, $\mathrm{NPBS}=$ non-polarizing beam splitter, GTP = Glan-Thompson prism, $\mathrm{PD}=$ photo diode, $\mathrm{LD}=$ laser diode, $\mathrm{ADC}=$ analog-to-digital, DAC = digital-to-analog converter, $\mathrm{PC}=$ computer.

\subsection{Inertial force correction method}

The experimental setup for measuring the electrical and mechanical responses of the force transducer against the impact load ${ }^{24)}$ is demonstrated in Figure 6. A conventional S-shaped strain-gauge-type force transducer, whose nominal force is $200 \mathrm{~N}$, is attached to the base.

The velocity of moving part $\mathbf{v}_{\mathbf{1}}$ is measured by the means of Interferometer-1; then the position $\boldsymbol{x}_{\mathbf{1}}$, acceleration $\boldsymbol{a}_{\mathbf{1}}$, and force acing on the mass $\boldsymbol{F}_{\text {mass }}$ are calculated. In the same way, the velocity of the sensing element of the force transducer $\mathbf{v}_{\mathbf{2}}$ is measured by the means of Interferometer, the position $\mathbf{x}_{\mathbf{1}}$ and the acceleration $\mathbf{a}_{\mathbf{2}}$ are calculated when got the velocity $\mathbf{v}_{\mathbf{2}}$. The electric response of the transducer is measured using the digital voltmeter (DVM). With the proposed method, the electrical and mechanical responses of force transducers against impact loads can be simultaneously evaluated. 


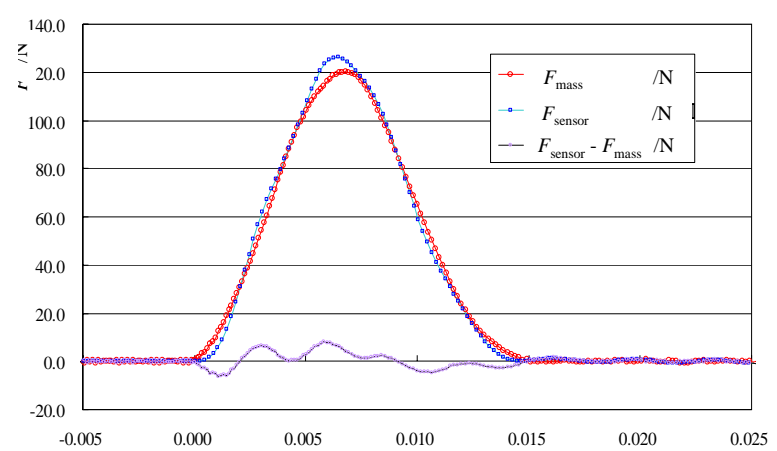

Figure 7. The force acting on the mass, $\boldsymbol{F}_{\text {mass }}\left(=\boldsymbol{F}_{\text {mass }}\right.$ $\boldsymbol{a}_{\text {mass }}$ ), the force calculated from the output signal of the force transducer and its calibration results $\boldsymbol{F}_{\text {sensor, }}$ and their difference $\boldsymbol{F}_{\text {sensor }}-\boldsymbol{F}_{\text {mass }}$.

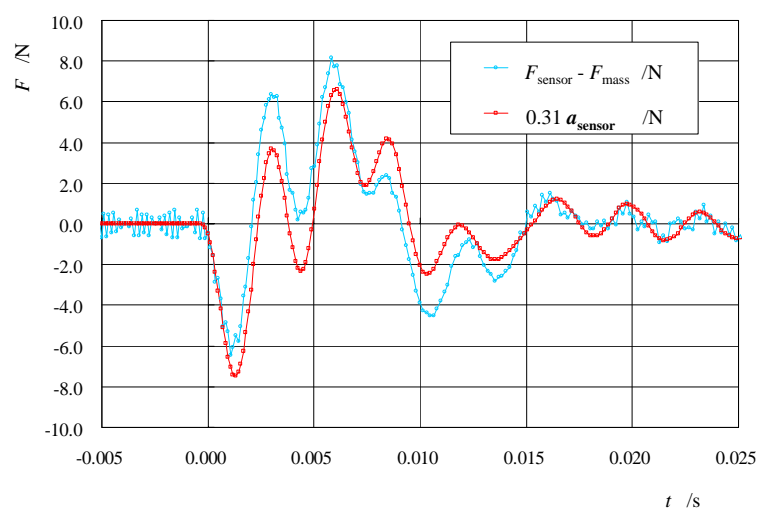

Figure 8. Difference between the values measured by the transducer and those measured by the proposed method, and the estimated inertial force of the sensor element.

Figure 7 shows the force measured by the force transducer and the proposed method. Recently, the authors found that the error in dynamic force measurements is almost proportional to the acceleration at the sensing point of the transducer; this can be explained as the effect of the inertial force of a part of the transducer itself ${ }^{24}$. From the relationship between the acceleration of the sensing point $\boldsymbol{a}_{2}$ and the difference between the values measured by the transducer and those measured by the proposed method, $\boldsymbol{F}_{\text {diff }}=\boldsymbol{F}_{\text {trans }}-\boldsymbol{F}_{\text {mass }}$, the regression line, $\boldsymbol{F}_{\text {reg }}$ $=\boldsymbol{F}_{\text {trans }}-\boldsymbol{F}_{\text {mass }}=0.325 \boldsymbol{a}_{\mathbf{2}}$, is estimated. The inclination of the line, 0.325 , can be considered as the estimated effective inertial mass of the transducer, $\boldsymbol{M}_{\text {estimated }}$.

Figure 8 depicts the difference between the values measured by the transducer and those measured by the proposed method, and the estimated inertial force of the sensor element. The two curves, $\boldsymbol{F}_{\text {diff }}=$ $\boldsymbol{F}_{\text {trans }}-\boldsymbol{F}_{\text {mass }}$ and $\boldsymbol{F}_{\text {reg }}=0.325 \boldsymbol{a}_{\mathbf{2}}$, coincide well with each other.

\section{Material Tester Based on the LMM}

With the similar setup given in Figure 2, the material tester without using force transducer can be realized. For example, if the force transducer shown in Figure 2 was replaced by a material, the impact response of a material can be evaluated.

If a small linear air bearing is used in the setup shown in Figure 2, dynamic force calibration with small force and/or material test with small force can be realized.

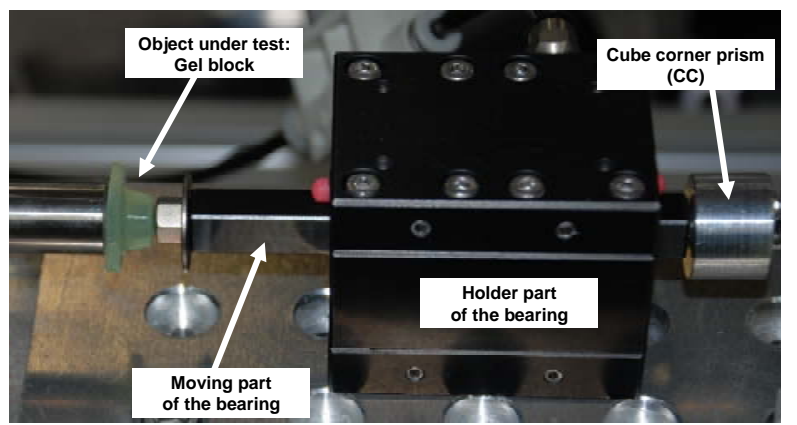

Figure 9. Photograph around the test section

Figure 9 shows a gel block tested under microforce based on the LMM. A pneumatic linear bearing is used to obtain linear motion with negligible friction acting on the mass, i.e., the piston-shaped moving part of the bearing. The impact force is generated and applied to the object under test by collision with the mass. An initial velocity is manually given to the moving part. A corner-cube prism (CC), that forms part of the interferometer, and a metal block with a round-shaped tip are attached to the moving part (made in aluminum with square pole shape); its total mass $\mathrm{M}$ is approximately $21.18 \mathrm{~g}$. The inertial force acting on the mass is measured highly accurately using an optical interferometer. The gel block is attached to the metallic base.

Figure 10 represents the data processing procedure in a collision experiment. During the experiment, the beat frequency, $\boldsymbol{f}_{\text {beat }}$, and the rest frequency, $\boldsymbol{f}_{\text {rest }}$, are measured highly accurately using an optical interferometer. The Doppler shift frequency is taken as the difference between the beat frequency and the rest frequency. The velocity, position, acceleration and inertial force of the mass are calculated from the time-varying Doppler shift frequency afterward. The velocity is proportional to the Doppler shift frequency. The acceleration is calculated by differentiating the velocity, while the inertial force of the mass is calculated by $\boldsymbol{F}=\boldsymbol{m a}$. The position is calculated by integrating the velocity. 


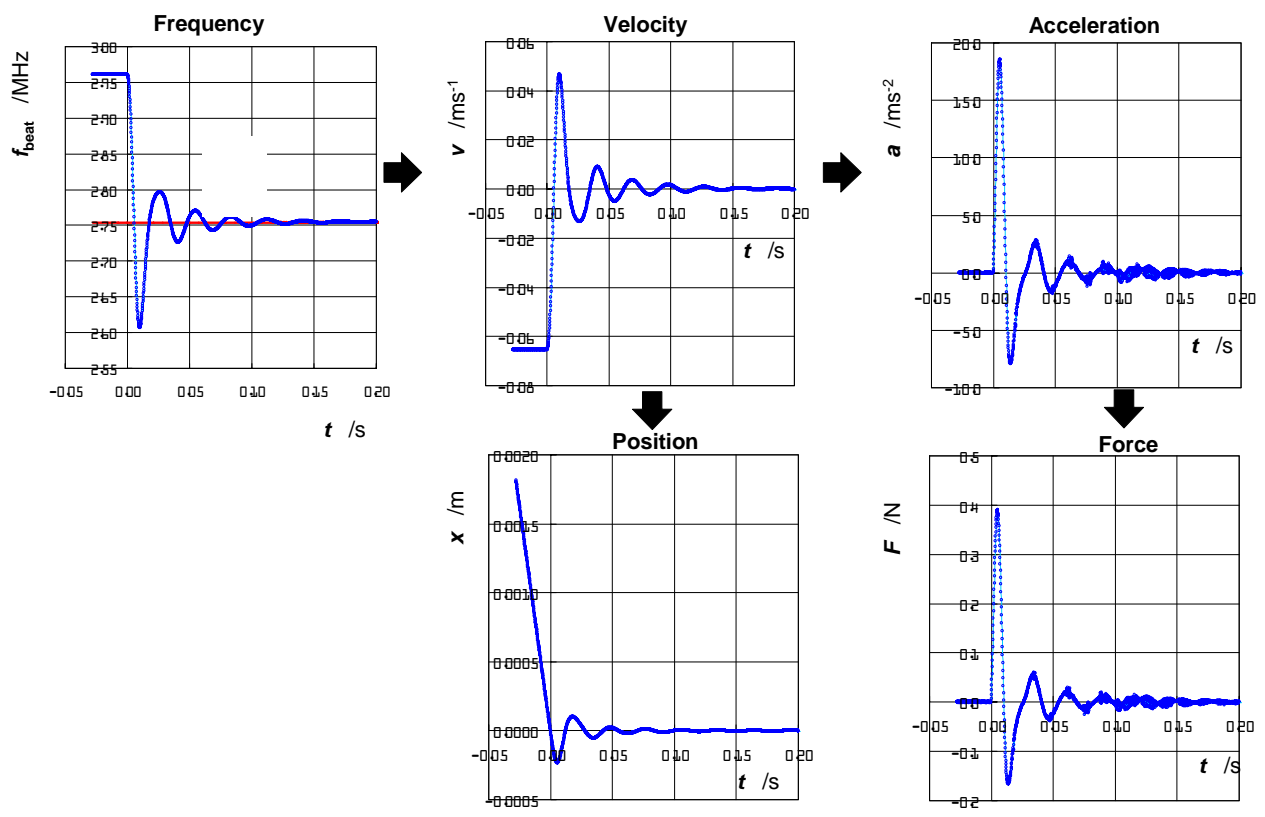

Figure 10. Data processing procedure: calculation of velocity, acceleration and force from frequency.

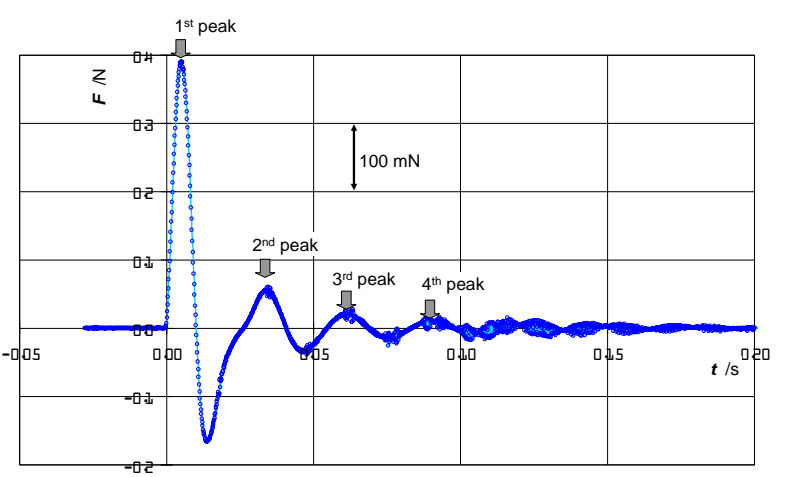

Figure 11. Measured force against time

Figure 11 gives the change in force acting on the mass from the gel block, $\boldsymbol{F}=\boldsymbol{M a}$, against time. In the collision experiment shown in Figure 11, the mass did not apart from the gel block after the first collision due to the adhesiveness of the gel block under test. The maximum value of the impact force, $\boldsymbol{F}_{\mathbf{m a x}}$, is approximately $390 \mathrm{mN}$ and the half width at half maximum $\boldsymbol{W}_{\mathbf{h}}$ is approximately $8.1 \mathrm{~ms}$.

While, materials usually change with temperature and environment. In those cases, the author is planning building a constant temperature box and it includes material and hit part of moving part. Figure 12 describes a setup of a material tester based on the LMM, which is planning to be developed.

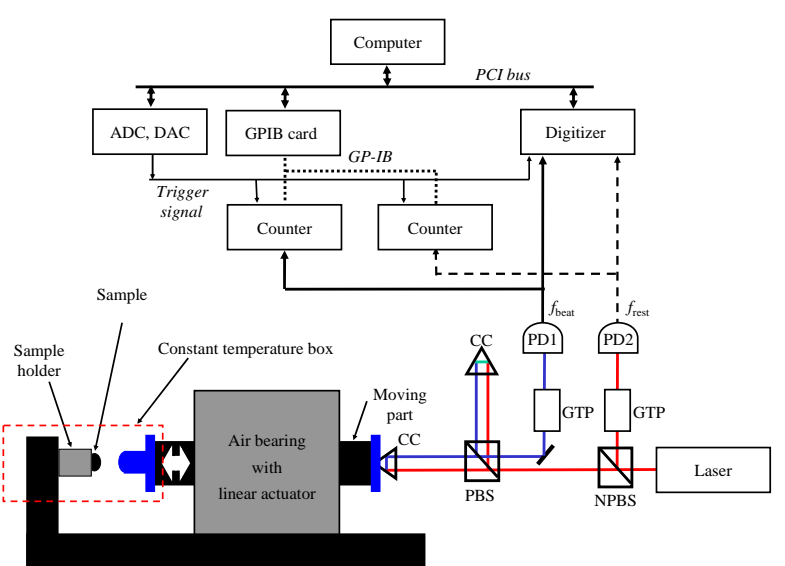

Figure 12. Material tester with impact force.

The features of the instrument are as follows:

1) Air bearing with liner motor: To realize the arbitrary setting of the initial velocity of the levitated mass, a liner motor is embedded in a pneumatic linear bearing.

2) Constant temperature box: To realize the arbitrary setting of the temperature of the material under test, a constant temperature box is introduced.

3) High precision measurement of the Doppler shift frequency: The frequency appearing at the out port of the optical interferometer PD is recorded using a digitizer, then the frequency calculated from the digitized waveform. Using the developed method, both the resolution and the sampling rate can be significantly improved.

\section{Space Scale}

With similar principle of LMM used to measure inertial force, the author also proposed a design for a 
body mass measurement device (BMMD), which called "Space Scale"14,17).

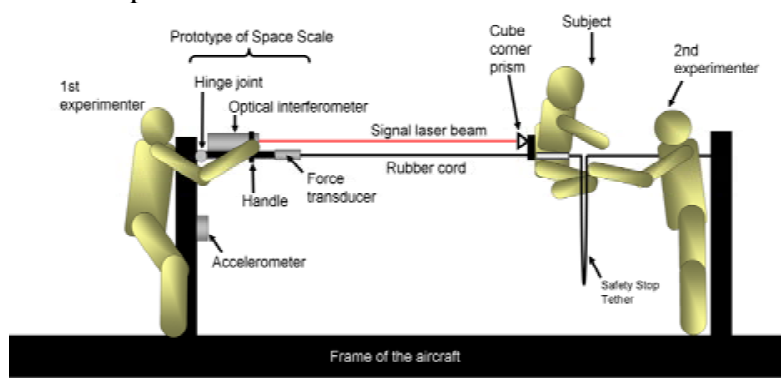

Figure 13. Experiment setup for human mass measurement.

A parabolic flight experiment setup of Space Scale is shown in Figure 13. Accelerometer was used to measure the aircraft's acceleration along moving direction, in order to calibrate the velocity relation to inertial system. The $1^{\text {st }}$ experimenter held on prototype of space scale and made sure that the laser focused on the cube corner prism. The subject body was levitated and stabilized by $2^{\text {nd }}$ experimenter. The rubber cord was used to supply force which made subject moving and measured by force transducer. The velocity of subject was precisely measured by using interferometer and then the acceleration of subject also been calculated. With force and acceleration, the mass of subject can be accurately worked out by using Newton's second law.
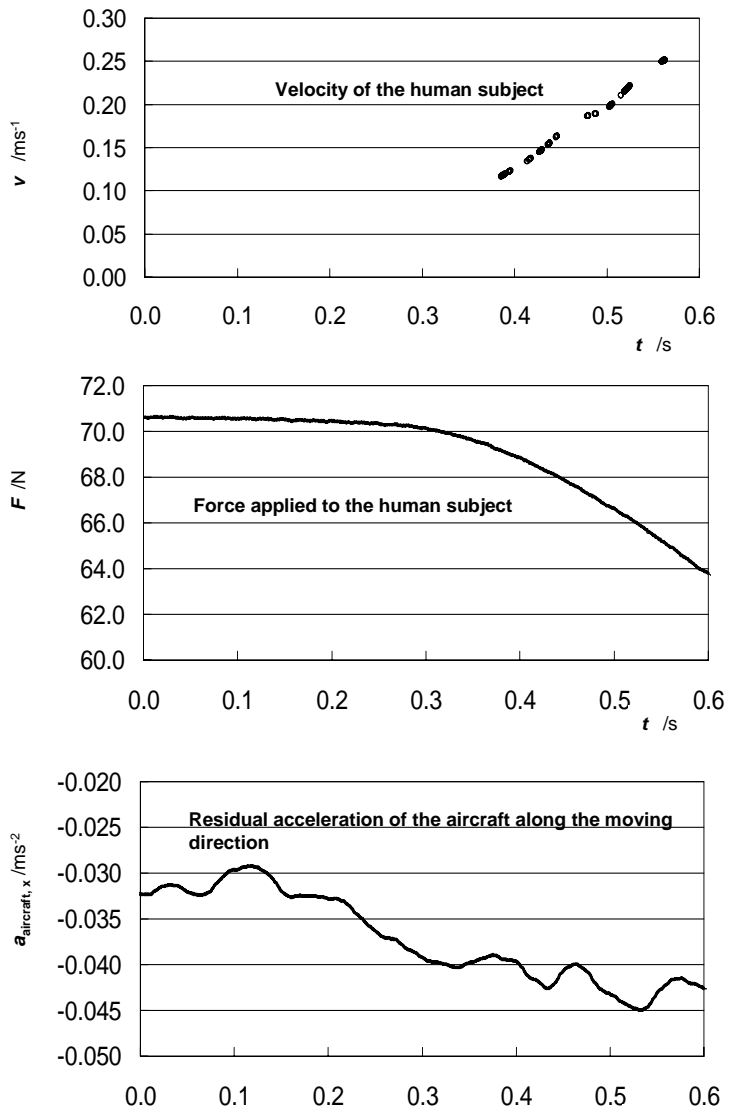

Figure 14. Velocity $v$ of the subject measured using the optical interferometer, force $\boldsymbol{F}$ applied to the subject measured using the force transducer, and residual acceleration of the aircraft $a_{\text {aircraft }}$ along the mass movement direction measured using the eccelerometer.

In Figure 14, 248 plots of velocity are obtained. The first plot was $(\mathrm{t} 1, \mathrm{v} 1)=(0.385 \mathrm{~s}, 0.116 \mathrm{~m} / \mathrm{s})$, and the final plot was $(\mathrm{t} 2, \mathrm{v} 2)=(0.563 \mathrm{~s}, 0.252 \mathrm{~m} / \mathrm{s})$. The velocity change during the period between $\mathrm{t} 1$ and $\mathrm{t} 2$ is $0.136 \mathrm{~m} / \mathrm{s}$. In cording to calibrate the velocity of subject human relation to inertial system. The velocity change of floor of aircraft along the cabin's longitudinal direction $\Delta v_{\text {aircraft }}=\int_{t_{2}}^{t_{1}} a_{\text {aircraft }} d t=-7.5 \times 10^{-3} \mathrm{~m} / \mathrm{s}$ is $5.5 \%$ corresponded to the human velocity change $0.13 \mathrm{~m} / \mathrm{s}$. Therefore, the human velocity $v_{\text {inertial }}$ changed related to inertial system is $0.129 \mathrm{~m} / \mathrm{s}$. The force $F$ ( $F=\int_{t_{2}}^{t_{1}} \int F d t$ ) applied on huam is $11.9 \mathrm{~N}$. So the mass of human is estimated as $M_{\text {mass }}=\int F d t / v_{\text {inertial }}=92.2 \mathrm{~kg}$. The measurement error was calculate as $M_{\text {mass }} / M_{\text {cal }}-1=-0.11$, where $M_{c e l}$ means the mass of human which has measured before experiment.

In figure 14 , the velcoity of subject only got during 0.116 s to $0.563 \mathrm{~s}$ in parabolic flight experiment. It's because that the subject can not keep moving linear and then laser can not focuse on the cube corner prism. In order to calculate the rotation of subject and modify the velcoity which calculated by interferometer, the authors have using to camera to record the experimental process and using image segmentation technology $^{39)}$ and camera calibration ${ }^{40)}$ to calculate the velocity, $\mathbf{v}_{\text {camera }}$, of subject.

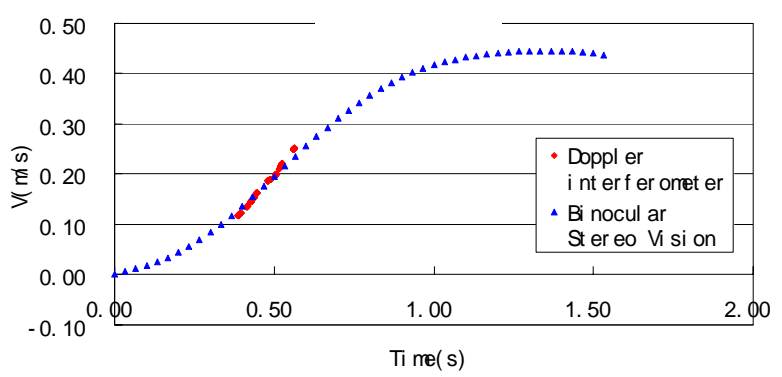

Figure 15. Velocity data measured by Doppler inferometer and binocular stereo vision compared.

Figure 15 shows the velocity compare between using two kind of method, space scale and binocular stereo vision. The RMS (root-mean-square) value of errors which are between two kinds data is $0.0022 \mathrm{~m} / \mathrm{s}$. The relative standard uncertainty of optical interferometer is $0.0003 \mathrm{~m} / \mathrm{s}$. Therefore, the standard uncertainty in measuring the velocity is estimated to be $0.0024 \mathrm{~m} / \mathrm{s}$. That means two kinds of methods coincide well and the data of blue one can be used to calculate 
the rotation which is the future work of subject during expriment.

\section{Discussions}

1) For dynamic calibration, the possible causes for the difference between of the output electric signal of strain-gauge-type force sensors the static and dynamic characteristics can be described as following:

1. Inertial mass: the effect of the inertial force of the mass which as a part of the sensor.

2. Strain gauge: the difference between the static and dynamic characteristics of the strain gauge.

3. Elastic body: the difference between the static and dynamic characteristics of the elastic body.

4. Signal processor: the difference between the static and dynamic characteristics of the electric signal processing system.

In the experiment using the S-shaped strain-gauge-type force sensor, the effect of the inertial mass was dominant. As shown in Figure 6, the simple spring-mass model explained the difference between the static and dynamic characteristics' output electric signal well.

However, some discrepancies are still observed. This indicates that there are still have some improvement rooms. For example, if a more sophisticated mechanical model, such as a finite element model, is adopted and the distribution of the acceleration and inertial force inside of the sensor are considered, the accuracy of the correction might be improved.

For accurate measurement, the force sensor error should be corrected. In this case, the accelerometer could be attached to the S-shaped strain-gauge-type force sensor which used in the experiment. Then the correction for the dynamic measurement error caused by the effect of inertial force acting on a part of the sensor structure itself can be done using the output signal of the accelerometer attached.

Since the output signal of the force sensor is thought to be almost proportional to the deformation of the sensor structure, and then the acceleration of the sensing point of the sensor is proportional to differential coefficient of second order of the output signal of the force sensor. Therefore, the correction for effect of inertial force acting on a part of the sensor structure itself could be done using only the output signal of the force sensor itself.

A design for realizing a low cost instrument is now also planning to be developed. In this case, a laser diode is used instead of a Zeeman-type two-frequency He-Ne laser and a pendulum is used instead of a pneumatic linear bearing.

The author would like to develop the following technologies and make them being commercialized.
Dynamic calibration method for force transducers: With this, the correction and the uncertainty evaluation of the measured forces with arbitrary waveform can be possible.

High precision material tester based on the LMM: In the tester, neither a force transducer nor a position sensor is used. Force is calculated according to its definition, which is the product of mass and acceleration. The acceleration is calculated from the mass's velocity which only computed from the Doppler shift frequency.

Thus, the stability of laser wave is very important. The authors ${ }^{35,37,38)}$ have improved some wavelength-insensitive laser. The purpose of these methods is to make sure the wavelength more insensitive and improve the Doppler shift frequency more accurately.

2) For material tester based on LMM, as shown in figure 12, the materials tester consists of a linear motion guide of the mass and an optical interferometer.

As the linear motion guide, a small aerostatic linear bearing is used in the experiment. The friction acting inside the aerostatic bearing is very small and the accuracy of its motion is very precise. However, it is expensive and requires the compressed air. It cannot be used in the high-temperature, the low-temperature or the vacuumed environments. If the pendulum mechanism developed ${ }^{28)}$ is used instead of the aerostatic linear bearing, measurement under high-temperature, low-temperature or vacuumed environments will be possible. The introduction of an electromagnetic linear bearing will also be effective for some purposes.

As the single-frequency measurement means for the optical interferometer, a low-cost digitizer with the Zero crossing Averaging Method (ZAM) ${ }^{29,30)}$ has been improved. In the LMM, the Doppler frequency shift of the laser light reflecting on the target is measured to determine the velocity of the inertial mass. Because the velocity of the rigid object takes one value at one time, the resulted Doppler frequency shift takes one value at one time.

3) For space scale, The flight prototype Space Scale was developed, and its efficiency was confirmed by evaluating the mass of the human subject in the parabolic flight experiment. A prominent feature of the proposed instrument is the use of a bungee cord as the source of force. This results in the following significant advantages.

1. A simple, lightweight, and compact structure of the instrument: It requires no actuator, and it can be stored in a small space when not deployed for use.

2. Strong and gradually-changing force: It requires only the initial tensional force made by a human subject.

3. Elimination of other external forces: Since the human subject is floated in space during the measurement, the mass is free from any other 
external forces, such as friction due to mechanical contact with other materials.

4. Large displacement during the measurement: This reduces the effect of change in subject posture (i.e., change in the density distribution of the astronaut). For example, if translational displacement during the measurement becomes 10 times larger, then the effect of the change of the posture relative to the center of gravity of the subject becomes 1 part of 10 (1/10).

5. Combined work with camera will effectively to describe subject's position, velocity, acceleration and rotation.

\section{Conclusions}

Dynamic error measurement methods of force sensors toward the dynamic calibration and correction have been reviewed. The methods are based on the Levitation Mass Method (LMM). In the LMM, the inertial force of a mass levitated using a pneumatic linear bearing is used as the reference force applied to the objects under test, such as force transducers, materials or structures. The inertial force of the levitated mass is measured using an optical interferometer. In this paper, software for correcting the dynamic error of force sensors has been proposed.

\section{Acknowledgment}

This work was supported by the Grant-in-Aid for Scientific Research (B) 19360185 (KAKENHI 19360185) and the research-aid fund of the Asahi Glass Foundation.

\section{References}

1. Y. Fujii, Measurement of Steep impulse Response of a Force Transducer, Meas. Sci. Technol., 14(1), 65-69, 2003.

2. Y. Fujii, A Method for Calibrating Force Transducers Against Oscillation Force, Meas. Sci. Technol., Vol. 14(8), 1259-1264, 2003.

3. Y. Fujii, Proposal for a step response evaluation method for force transducers, Meas. Sci. Technol., 14(10), 1741-1746, 2003.

4. Y. Fujii, Dynamic Force Calibration Methods for Force Transducers, IEEE Trans. Instrum. Meas., 58(7), 2358-2364, 2009.

5. Y. Fujii, Toward Dynamic Force Calibration, Measurement, 42(7), 1039-1044, 2009.

6. Y. Fujii and T. Yamaguchi, Method for Evaluating Material Viscoelasticity, Rev. Sci. Instrum., 75(1), 119-123, 2004.

7. Y. Fujii and T. Yamaguchi, Proposal for Material Viscoelasticity Evaluation Method Under Impact Load, J. Mater. Sci., 40(18), 4785 - 4790, 2005.

8. Y. Fujii and D.W. Shu, Impact Force Measurement of an Actuator Arm of a Hard Disk Drive, Int. J. Impact Eng., 35(2), 980108, 2008.
9. Y. Fujii and T. Yamaguchi, Optical Method for Evaluating Material Friction, Meas. Sci. Technol., 15(10), 1971-1976, 2004.

10. Y. Fujii, Method for Measuring Transient Friction Coefficients for Rubber Wiper Blades on Glass Surface, Tribology International, 41(1), 17-23, 2008.

11. Y. Fujii, T. Yamaguchi and J. Valera, Impact Response Measurement of a Human Arm, Exp. Techniques, 30(3), 64-68, 2006.

12. Y. Fujii and T. Yamaguchi, Method of Evaluating the Force Controllability Of Human Finger, IEEE Trans. Instrum. Meas., 55(4), 1235-1241, 2006.

13. Y. Fujii and K. Maru, Method for Evaluating the Electrical and Mechanical Characteristics of a Voice Coil Actuator, Precision Engineering, (in press).

14. Y. Fujii and K. Shimada, Instrument for Measuring the Mass of an Astronaut, Meas. Sci. Technol., 17(10), 2705-2710, 2006.

15. Y. Fujii and K. Shimada, The Space Scale: an Instrument for Astronaut Mass Measurement, Trans. Jpn. Soc. Aeronaut. Space Sci., 50(170), 251-257, 2008.

16. Y. Fujii et al, Mass Measuring Instrument for use Under Microgravity Conditions, Rev. Sci. Instrum., 79(5), 056105-1-3, 2008.

17. Y. Fujii, K. Shimada and K. Maru, Instrument for Measuring the Body Mass if Astronauts Under Microgravity Conditions, Microgravity Sci. Tec., 22(1), 115-121, 2010.

18. Y.Fujii et al., Instrument for Measuring the Body Mass of Astronaut, Trans. Jpn. Soc. Aeronaut. Space Sci. Space Technol. Jpn., (in press).

19. Y. Fujii, Optical Method for Accurate Force Measurement: Dynamic Response Evaluation of an Impact Hammer, Opt. Eng., 45(2), 023002-1-7, 2006.

20. Y. Fujii, Method for generating and measuring the micro-Newton level forces, Mech. Syst. Signal Pr., 20(6), 1362-1371, 2006.

21. Y. Fujii, Microforce Materials Tester, Rev. Sci. Instrum. 76(6), 065111-1-7, 2005.

22. Y. Fujii, Microforce Materials Tester Based on the Levitation Mass Method, Meas. Sci. Technol., 18(6), 1678-1682, 2007.

23. Y. Fujii, Method of Generating and Measuring Static Small Force Using Down-Slope Component of Gravity, Rev. Sci. Instrum., 78(6), 066104-1-3, 2007.

24. Y. Fujii, Measurement of Force Acting on s Moving Part of a Pneumatic Linear Bearing, Rev. Sci. Instrum., 74(6), 3137-3141, 2003.

25. Y. Fujii, Frictional Characteristics of an Aerostatic Linear Bearing, Tribol. Int., 39:9, 888-896, 2006.

26. Y. Fujii, An Optical Method for Evaluating Frictional Characteristics of Linear Bearings, Opt. Lasers Eng., 42(5), 493-501, 2004. 
27. Y. Fujii and K. Maru, Optical Method for Evaluating Dynamic Friction of a Small Linear Ball Bearing, Tribol. T., 53(2), 169-173, 2010.

28. Y. Fujii, Pendulum for Precision Force Measurement, Rev. Sci. Instrum., 77(3), 035111-1-5, 2006.

29. Y. Fujii and J. Valera, Impact Force Measurement Using an Inertial Mass and a Digitizer, Meas. Sci. Technol., 17(4), 863-868, 2006.

30. Y. Fujii, Impact Response Measurement of an Accelerometer, Mech. Syst. Signal Pr., 21(5), 2072-2079, 2007.

31. Y. Fujii and J. P. Hessling, A Frequency Estimation Method for use in the Levitation Mass Method, Exp. Techniques, 33(5), 64-69, 2009.

32. Y. Fujii, Measurement of the Electrical and Mechanical Responses of A Force Transducer Against Impact Forces, Rev. Sci. Instrum., 77(8), 085108-1-5, 2006.

33. Y. Fujii, Method for Correcting the Effect of the Inertial Mass on Dynamic Force Measurements, Meas. Sci. Technol., 18(5), N13-N20, 2007.

34. Fujii and K. Maru, Self-Correction Method for Dynamic Measurement Error of Force Sensors, Exp. Techniques, (in press, published online).

35. K. Maru and Y. Fujii, Wavelength-Insensitive Laser Doppler Velocimeter using Beam Position
Shift Induced by Mach-Zehnder Interferometers, Opt. Express, 17(20), 17441-17449, 2009.

36. K. Maru and Y. Fujii, Reduction of Chromatic Dispersion Due to Coupling for Synchronized-router-based flat-passband Filter using Multiple-input Arrayed Waveguide Grating, Opt. Express, 17(24), 22260-22270, Nov. 2009.

37. K. Maru and Y. Fujii, Integrated Wavelength-Insensitive Differential Laser Doppler Velocimeter using Planar Lightwave Circuit, J. Lightwave Technol., 27(22), 5078-5083, Nov. 2009.

38. K. Maru, K. Kobayashi, and Y. Fujii, Multi-Point Differential Laser Doppler Velocimeter Using Arrayed Waveguide Gratings with Small Wavelength Sensitivity, Opt. Express, 18(1), 301-308, Jan. 2010.

39. Jin Tao et al., Extracting a Circle and its Center in a MOVING DUMMY MASS, Electronics World, 116, 22-25, 2010.

40. Jin Tao et al., VNIR Image Simulation Based on Low-level Flight Image Data, Chinese Optical Letters, 8(6), 601-605, 2010. 